Research Article

\title{
Impact of the Italian Society of Anatomic Pathology and Diagnostic Cytology Classification of Thyroid Nodules in the Treatment of Indeterminate Follicular Lesions: Five-Year Results at a Single Center
}

\author{
M. Pastoricchio $\mathbb{D},{ }^{1}$ A. Cubisino, ${ }^{1}$ A. Lanzaro, ${ }^{1}$ M. Troian $\left(\mathbb{D},{ }^{1}\right.$ F. Zanconati, ${ }^{2}$ S. Bernardi $\left(\mathbb{D},{ }^{2,3}\right.$ \\ B. Fabris, ${ }^{2,3}$ N. de Manzini $\mathbb{D}^{1},{ }^{1}$ and C. Dobrinja ${ }^{1}$ \\ ${ }^{1}$ Division of Clinical Surgery, Department of Medical, Surgical and Health Sciences, University of Trieste, \\ Cattinara Teaching Hospital, Trieste 34149, Italy \\ ${ }^{2}$ Department of Medical, Surgical and Health Sciences, Università Degli Studi di Trieste, Cattinara Teaching Hospital, \\ Strada di Fiume 447, 34149 Trieste, Italy \\ ${ }^{3}$ Endocrinology Unit-Azienda Sanitaria Universitaria Integrata Trieste, Cattinara Teaching Hospital, Strada di Fiume 447, \\ 34149 Trieste, Italy \\ Correspondence should be addressed to M. Pastoricchio; manuela.pastoricchio@gmail.com
}

Received 16 November 2019; Revised 3 March 2020; Accepted 9 March 2020; Published 14 April 2020

Academic Editor: A. Lania

Copyright (C) 2020 M. Pastoricchio et al. This is an open access article distributed under the Creative Commons Attribution License, which permits unrestricted use, distribution, and reproduction in any medium, provided the original work is properly cited.

\begin{abstract}
Purpose. Aim of the study was to assess the impact of the Italian Society of Anatomic Pathology and Diagnostic Cytology (SIAPEC) classification of 2014, on the treatment of indeterminate thyroid lesions (TIR3). Methods. We retrospectively analyzed patients undergoing thyroid surgery for TIR3 lesions between 2013 and 2018, at the General Surgery Department of Trieste University Hospital. According to the SIAPEC classification, patients were divided into TIR3A and TIR3B groups. All patients treated before 2014 underwent surgical treatment, and surgical specimens were retrospectively classified after revision of fineneedle aspiration cytology. Starting 2014, TIR3A patients were treated only when symptomatic (i.e., coexistent bilateral thyroid goiter or growing TIR3A nodules), whereas TIR3B patients always received surgical treatment. Hemithyroidectomy (HT) was the procedure of choice. Total thyroidectomy (TT) was performed in case of concurrent bilateral goiter, autoimmune thyroid disease, and/or presence of BRAF and/or RAS mutation. Lastly, we analyzed the malignancy rate in the two groups. Results. 29 TIR3A and 90 TIR3B patients were included in the study. HT was performed in 10 TIR3A patients and 37 TIR3B patients, respectively, with need for reoperation in 4 TIR3B $(10.8 \%)$ patients due to histological findings of follicular thyroid carcinoma $>1 \mathrm{~cm}$. The malignancy rates were $17.2 \%$ in TIR3A and $31.1 \%$ in TIR3B, $(p=0.16)$. Predictability of malignancy was almost $89 \%$ in BRAF mutation and just $47 \%$ in RAS mutation. Conclusions. The new SIAPEC classification in association with biomolecular markers has improved diagnostic accuracy, patient selection, and clinical management of TIR3 lesions.
\end{abstract}

\section{Introduction}

Thyroid nodules have become a common finding in clinical practice, being detected in up to $67 \%$ of general population due to widespread use of sensitive imaging techniques [1-5]. Although most nodules are benign, thyroid cancer is detected in up to $5-15 \%$ of cases depending on several risk factors (e.g., age, sex, family history, and previous radiation exposure) [6-8]. The most common malignancy is differentiated thyroid cancer (DTC), which includes papillary and follicular histologies and represents the vast majority $(>90 \%)$ of all thyroid cancers [9].

According to current guidelines, management is primarily based on morphologic classification of cytologic 
samples obtained by fine-needle aspiration (FNA), complemented by clinical and imaging findings, and increasingly supplemented by molecular markers test results [10-13]. Various classification schemes based on thyroid cytology have been proposed and their validity has been extensively demonstrated [10-13]. However, approximately $25 \%$ of all FNAs will fall into an indeterminate category, which represents a difficult challenge for clinicians since malignancy, although relatively low $(20-30 \%)$, cannot be safely excluded [14-18]. FNA cytology is particularly unreliable in differentiating between benign and malignant follicular thyroid disease as the specific characteristics of aggressiveness (i.e., capsular and/or vascular invasion) can be established only after thorough histopathologic examination $[15,18]$. As a result, most patients with indeterminate cytology at FNA biopsy will require diagnostic surgery, but only $20 \%$ of cases will turn out to be malignant, leading to more than $80 \%$ of unnecessary surgery excisions with associated risks and costs [14-18].

Classification systems have been trying to address the issue of reducing unneeded surgery without missing potentially malignant nodules by subdividing the indeterminate category into two different classes based on architectural features, grade of nuclear atypia, and relative risk of malignant occurrence (Table 1). In 2007, the United States National Cancer Institute (NCI) proposed the Bethesda System for Reporting Thyroid Cytology (BSRTC), in which indeterminate nodules were divided in class III or AUS/FLUS (atypia of undetermined significance or follicular lesion of undetermined significance) and class IV or FN/ SFN (follicular neoplasms or suspicious for a follicular neoplasm), with different expected malignancy rates and management options [19]. Similarly, in 2009, the Royal College of Pathologists (RCPath) revised the British Thyroid Association (BTA) guidelines and divided the indeterminate THY3 category into THY3a (atypia/nondiagnostic) and THY3f (follicular lesion/suspect follicular neoplasia) [20]. More recently, the Italian Society of Anatomic Pathology and Diagnostic Cytology (SIAPEC) modified the previous classification of TIR3 indeterminate lesions by introducing two different subclasses, namely, TIR3A (low-risk indeterminate), for which clinical and radiological follow-up may be adequate, and TIR3B (high-risk indeterminate), for which surgery is always required [21,22]. It has to be noted that, unlike the BSRTC and BTA-RCPath systems, the 2014 SIAPEC classification extended the TIR3B category to include those cases with mild or focal nuclear atypia suggestive of papillary carcinoma, which are expected to have a higher risk of malignancy $[13,21,22]$. Anyway, estimated rates of cancer are less than $10 \%$ for TIR3A and $15-30 \%$ for TIR3B, which are essentially similar to those reported by the BSRTC and BTA/RCPath classifications (i.e., $5-15 \%$ rates for the AUS/FLUS and THY3a categories and $15-30 \%$ for the FN/ SFN and THY3f categories, respectively) $[19,20]$.

Several studies evaluating the BSRTC and BTA/RCPath classification systems have reported higher than expected malignancy rates $[23,24]$, whereas a more accurate estimate of the risk of malignancy for the indeterminate lesions have been observed with the adoption of the 2014 SIAPEC classification [25-28].
The aim of the present study was to assess the clinical impact of the 2014 SIAPEC classification on the management of indeterminate thyroid lesions, particularly with regard to malignancy rates and care strategies, including the role of surgery as a treatment option and the diagnostic impact of molecular markers in predicting malignancy.

\section{Methods}

We performed a retrospective analysis of all surgical procedures performed for thyroid disease at the General Surgery Department of Trieste University Hospital between January 2013 and January 2018. All patients with solitary thyroid nodules and preoperative cytological diagnosis of indeterminate follicular lesion were enrolled in the study and assigned to two independent groups, namely, TIR3A and TIR3B, according to the 2014 SIAPEC classification. For those treated before the introduction of the new system classification, subdivision was performed after retrospective revision of FNA cytology. Of these, the vast majority of patients fell into the TIR3B category.

Preoperative work-up of thyroid nodules consisted of full clinical examination and ultrasonography (US) of the neck, followed by FNA when nodules were $>1 \mathrm{~cm}$ of diameter and/or presented US features of malignancy (e.g., solid composition, hypoechogenicity, irregular margins, microcalcifications, and cervical lymph node involvement). The US classification was obtained for all thyroid nodules according to the thyroid imaging reporting and data system (TIRADS) [10, 11, 29-31]. Based on US appearance, we distinguished two major categories: nodules presenting with a low to intermediate US risk of malignancy (TIRADS 3, 4a, and $4 \mathrm{~b}$ ) and those with a high risk of malignancy (TIRADS $4 \mathrm{c}$ and 5). TIR3A patients underwent clinical and instrumental follow-up, including neck US. The US appearance of the nodules and their initial size, in many cases, oriented the management strategy of these patients with consequent inclusion to surgery or to follow-up.

All FNAs were performed under US guidance by an experienced radiologist. Smears were either fixed in alcohol and stained by Papanicolaou stain or air dried and stained with Giemsa stain. Starting 2014, all TIR3B lesions and specific TIR3A nodules (i.e., those growing in size and those with US features of malignancy) are tested for BRAF mutation. From 2015, molecular testing includes RAS mutation assessment as well [32-35].

The material was obtained after digitization of the cytological specimen to define the gene structure for detecting the main and frequent mutations that are thyroid related. The molecular biology survey was performed on DNA extracted from cytological material aspirated by nodule of the thyroid gland and was conducted by real-time PCR technique after extraction with QIAamp DNA Mini Kit. The analysis detected the main mutations of codons 594, 600, and 601 as concerns BRAF and of codons 12, 13, 59, 61, 117, and 146 as concerns RAS (NRAS, KRAS, and HRAS). The quality of the laboratory test was submitted by the Associazione Italiana di Oncologia Medica (AIOM)-SIAPEC control. Particularly in TIR 3A, the mutational status of RAS 
TABLE 1: Comparison of the Bethesda, the RCPath, and the SIAPEC diagnostic categories.

\begin{tabular}{|c|c|c|}
\hline BSRTC & RCPath & SIAPEC \\
\hline I. Nondiagnostic or unsatisfactory & THY1/THY1c. Nondiagnostic /Cystic & $\begin{array}{l}\text { TIR1. Nondiagnostic } \\
\text { TIR1C: Nondiagnostic } \\
\text { cystic }\end{array}$ \\
\hline II. Benign & THY2/THY2c. Nonneoplastic & $\begin{array}{c}\text { TIR2: Nonmalignant } \\
\text { /benign }\end{array}$ \\
\hline $\begin{array}{l}\text { III. Atypia of undetermined significance (AUS) or } \\
\text { follicular lesion of undetermined significance } \\
\text { (FLUS) }\end{array}$ & $\begin{array}{l}\text { THY3a. Neoplasm possible. Atypical features present but } \\
\text { not enough to place into any of the other categories }\end{array}$ & $\begin{array}{l}\text { TIR3A. Low-risk } \\
\text { indeterminate lesion }\end{array}$ \\
\hline $\begin{array}{l}\text { IV. Follicular neoplasm or suspicious for a follicular } \\
\text { neoplasm }\end{array}$ & $\begin{array}{c}\text { THY3f. Neoplasm possible-suggesting follicular } \\
\text { neoplasm }\end{array}$ & $\begin{array}{c}\text { TIR3B. High-risk } \\
\text { indeterminate lesion }\end{array}$ \\
\hline V. Suspicious for malignancy & THY4. Suspicious of malignancy & $\begin{array}{l}\text { TIR4. Suspicious of } \\
\text { malignancy }\end{array}$ \\
\hline VI. Malignant & THY5. Malignant & TIR5. Malignant \\
\hline
\end{tabular}

gene provided us the most useful information to guide the management decision.

The molecular markers were investigated in all TIR 3B patients and in a fraction of TIR3A patients. The reason driving the decision to perform the molecular analysis just in some TIR3A nodules was to investigate if the presence of such mutations could justify the growing in size of these nodules, thus prompting the surgical treatment instead of the follow-up suggested in these patients. Moreover, the presence of such mutations was helpful in guiding the most appropriate surgical strategy.

Management options are based on FNA cytology, according to the 2014 SIAPEC classification and the American Thyroid Association (ATA) guidelines $[10,13,22,30]$. Therefore, TIR3A patients underwent clinical and instrumental follow-up, consisting of serial medical evaluations performed by an endocrinologist, neck US, and laboratory testing. Surgery was considered only for symptomatic nodules, coexisting bilateral thyroid goiter, nodules growing in size and/or US features of malignancy. Hemithyroidectomy (HT) was the procedure of choice for solitary nodules, whereas total thyroidectomy (TT) was performed in case of bilateral thyroid goiter or in the presence of BRAF/RAS mutation, where available.

As far as TIR3B lesions are concerned, all patients underwent surgery. HT was the procedure of choice in case of intraparenchymal nodules with no known risk factors (i.e., negative BRAF/RAS status on FNA cytology, no history of previous head and neck irradiation, and no clinical nor radiological evidence of either nodal disease or distant metastases). TT was selectively proposed in the following cases: coexisting bilateral goiter, autoimmune thyroid disease, presence of BRAF/RAS mutation, prior head and neck irradiation, and family history of thyroid malignancies.

According to ATA guidelines $[10,30]$, in case of intraoperative finding of lymph nodes suspicious for metastasis, either unilateral or bilateral central neck dissection (CND) and/or lateral neck dissection (LND) were performed. Whenever final histopathology diagnosed malignancy in HT specimen, completion thyroidectomy was performed in the presence of one or more of the following: carcinoma $>10 \mathrm{~mm}$ in size, multifocal disease with an overall sum of all lesions' diameters $>10 \mathrm{~mm}$, microscopic extrathyroid extension, and aggressive histologic features (i.e., tall-cell, columnar-cell, or diffuse sclerosing variants).

Histopathologic data (e.g. multifocality, aggressive features, extracapsular invasion, and lymph node metastases) were recorded for all patients. Noninvasive follicular thyroid neoplasms with papillary-like nuclear features (NIFTPs) were considered as nonmalignant, according to the reclassification introduced into the 2017 World Health Organization [36].

The malignancy rates were analyzed in both groups in order to evaluate whether the 2014 SIAPEC classification improved diagnostic accuracy and if its association with biomolecular markers could improve prediction of malignancy. Furthermore, histopathologic data were analyzed in order to determine whether completion thyroidectomy or total thyroidectomy were appropriate or should have been considered overtreatment.

2.1. Statistical Analysis. Categorical variables were assessed using Fisher's exact test or Chi-squared test, when appropriate. Continuous variables were evaluated by using Student's $t$-test or Mann-Whitney $U$-test, when appropriate. A $p$ value of less than 0.05 was considered to be statistically significant. All statistical analyses were performed using GraphPad software.

\section{Results}

According to preoperative FNA cytology, a total of 119 patients underwent surgery for TIR3 lesions between January 2013 and January 2018. Of these, 29 cases had TIR3A nodules and 90 cases had TIR3B nodules. Population characteristics, including size (major diameter) and US appearance of the nodules, are summarized in Table 2.

In the TIR3A group, 10 patients underwent HT and 19 patients underwent TT. In the TIR3B group, 37 patients underwent $\mathrm{HT}$ and 53 patients underwent TT. Of those with positive molecular mutations, 2 TIR3A patients and 2 TIR3B patients preferred to be treated with HT despite accurate information upon potential risks and benefits of complete thyroidectomy. On the other side, TT was required by 4 
TABle 2: Surgical treatment, demographic, and pathologic data.

\begin{tabular}{|c|c|c|c|}
\hline & TIR 3A $(n=29)$ & TIR 3B $(n=90)$ & $p$ value \\
\hline \multicolumn{4}{|l|}{ TIRADS score } \\
\hline 3, 4a, 4b (low-intermediate risk) & 26 & 76 & \\
\hline Benign/malignant & $22 / 4$ & $57 / 19$ & \\
\hline $4 c, 5$ (high risk) & 3 & 14 & \\
\hline Benign/malignant & $2 / 1$ & $5 / 9$ & \\
\hline Nodule major diameter, $\mathrm{mm}$ (range) ${ }^{*}$ & $23.4 \pm 14(4-60)$ & $29 \pm 18(4-80)$ & \\
\hline \multicolumn{4}{|l|}{ Type of surgery } \\
\hline $\mathrm{HT}$ & 10 & 37 & \\
\hline TT & 19 & 53 & \\
\hline \multicolumn{4}{|l|}{ Reasons for TT } \\
\hline Bilateral goiter & 12 & 28 & \\
\hline Autoimmune thyroid disease & 4 & 5 & \\
\hline Nodule growing in size & 3 & 7 & \\
\hline BRAF/RAS mutation & 9 & 12 & \\
\hline Prior head \& neck irradiation & 0 & 0 & \\
\hline Family history of thyroid cancer & 0 & 1 & \\
\hline Completion thyroidectomy & $0(0 \%)$ & $4(10.8 \%)$ & 0.56 \\
\hline Malignancy & $5(17.2 \%)$ & $28(31.1 \%)$ & 0.16 \\
\hline pTla & 4 & 17 & \\
\hline pT1b & 1 & 3 & \\
\hline pT2 & 0 & 5 & \\
\hline pT3 & 0 & 3 & \\
\hline Multifocality/TTs for cancer & $1 / 4(25 \%)$ & $5 / 26(19.2 \%)$ & Ns \\
\hline Unilobar & 0 & 0 & \\
\hline Bilobar & 1 & 5 & \\
\hline Extrathyroid invasion & 0 & $2(7.1 \%)$ & Ns \\
\hline Vascular invasion & 0 & $1(3.6 \%)$ & Ns \\
\hline Aggressive variants & 0 & 0 & - \\
\hline Lymph node metastases/lymph node dissections & $1 / 1$ & $0 / 2$ & - \\
\hline \multicolumn{4}{|l|}{ Morphology } \\
\hline Taller than wide & 4 & 17 & \\
\hline Oval/round shape & 25 & 73 & \\
\hline \multicolumn{4}{|l|}{ Margins } \\
\hline Irregular & 3 & 5 & \\
\hline Regular & 26 & 85 & \\
\hline \multicolumn{4}{|l|}{ Microcalcifications } \\
\hline Yes & 5 & 12 & \\
\hline No & 24 & 78 & \\
\hline \multicolumn{4}{|l|}{ Echogenicity } \\
\hline Hypoechogen & 6 & 44 & \\
\hline Nonhypoechogen & 23 & 46 & \\
\hline \multicolumn{4}{|l|}{ Echostructure } \\
\hline Solid & 21 & 77 & \\
\hline Mixed & 8 & 13 & \\
\hline
\end{tabular}

Note. Data are presented as mean \pm SD where applicable. TT, total thyroidectomy; HT, hemithyroidectomy; ns, nonsignificant. ${ }^{\bullet} 0$ cases of TIRADS 5 reported. *in bilateral goiter, size of the dominant nodule. 9 one case of benignity of TIR3B lesion and coexistent occult PTMC in the controlateral lobe.

patients although no thyroid goiter and/or known risk factors for malignancy were present.

Among TIR3A patients undergoing HT, none required completion thyroidectomy since final histological examination revealed benign nodules in 9 cases (including one case of NIFTP), whereas 1 patient presented with occult papillary thyroid microcarcinoma (PTMC) with no need for further surgery according to current guidelines [10]. Among TIR3A patients undergoing TT, malignancy was found in 4 patients, mostly in the form of PTMC (3 out of 4 cases,
75.0\%). Unilateral CND was performed in 1 case due to intraoperative suspect of lymph node involvement, later confirmed at final histopathology. No case presented either extracapsular or vascular invasion. One NIFTP was reported after TT.

Among TIR3B patients undergoing HT, completion thyroidectomy was required in $4(10.8 \%)$ patients because of histological finding of minimally or widely invasive follicular thyroid carcinoma (FTC) $>1 \mathrm{~cm}$, whereas $2(5.4 \%)$ patients presented occult PTMC with no need for further surgery 
according to current guidelines [10]. Among TIR3B patients undergoing TT, malignancy was found in 22 cases, mostly in the form of PTMC ( 15 out of 22 cases, 68.2\%). Bilateral CND was performed in 2 patients, but no metastases were found at final histopathology. Extracapsular invasion was found in 2 patients and 1 patient presented vascular invasion. Two NIFTPs were reported.

Overall, the malignancy rate was $27.7 \%$, with no significant differences between the two groups (17.2\% in TIR3A patients vs. $31.1 \%$ in TIR3B patients, $p=0.16$ ). PTMC was the most frequently recorded type of cancer in both set of patients, with a total prevalence of $63.6 \%$ (21 out of 33 cases). A total of $47 \mathrm{HT}$ were performed with no need for further operation in $43(91.5 \%)$ patients.

A total of $91(76.5 \%)$ patients underwent molecular testing on preoperative FNA cytologic samples. Of these, 48 patients were tested for both BRAF and RAS mutation, whereas 43 patients, who were operated between 2013 and 2015, were tested for BRAF mutation only, because-as stated before-the RAS test was not available at our institution until 2015. When considering BRAF testing alone, sensitivity was $29.6 \%$ (95\% CI. $13.7 \%-50.2 \%)$ and specificity was $98.4 \%$ (95\% CI. 91.6\%-99.9\%), with a positive predictive value (PPV) of $88.9 \%$ (95\% CI. 51.2\%-98.4\%), a negative predictive value (NPV) of $76.8 \%$ (95\% CI. $72.1 \%-80.9 \%$ ), and an accuracy rate of $78.0 \%$ (95\% CI. $68.1 \%-86.0 \%$ ), respectively. When considering RAS testing alone, sensitivity was $43.7 \%$ (95\% CI. 19.7\%-70.1\%) and specificity was $75.0 \%$ (95\% CI. 56.6\%-88.5\%), with a PPV of $46.7 \%$ (95\% CI. $27.9 \%-66.5 \%$ ), a NPV of $72.7 \%$ (95\% CI. 62.3\%-81.1\%), and an accuracy rate of $64.6 \%$ (95\% CI. $49.5 \%-77.8 \%)$, respectively. Data are summarized in Tables 3 and 4.

\section{Discussion}

FNA cytology is currently regarded as the primary and most cost-effective diagnostic tool for evaluating thyroid nodules, being able to differentiate between benign and malignant disease in up to $70-80 \%$ of cases [10-13]. However, sensitivity drops when analyzing follicular-patterned neoplasms and further evaluations are required, including possibly unnecessary surgery and costly additional testing. Despite the efforts made to improve diagnostic sensitivity and accuracy of cytologic classifications, the indeterminate thyroid nodule remains a matter of debate among pathologists, especially when considering diagnostic criteria and clinical impact of molecular marker [14-18].

The present study aimed to assess the clinical impact of the 2014 SIAPEC classification on the management of indeterminate thyroid lesions, particularly with regard to malignancy rates, diagnostic impact of molecular markers, and relative care strategies. According to our results, the malignancy rate in TIR3B patients was nearly doubled when compared to that of TIR3A patients $(31.1 \%$ vs. $17.2 \%$, respectively). Although not significant on statistical analysis $(p=0.16)$, this difference is in line with data from a recent meta-analysis, where cancer prevalence of TIR3A and TIR3B was $14 \%$ and $33 \%$, respectively, considering NIFTP as a
TABLE 3: BRAF status in TIR3 population.

\begin{tabular}{lccc}
\hline & Number of cases & Benign & Malignant \\
\hline BRAF- & 82 & 63 & 19 \\
BRAF+ & 9 & 1 & 8 \\
& 91 & 64 & 27 \\
\hline
\end{tabular}

nonmalignant entity [37]. These data support the validity of the 2014 SIAPEC classification in cancer risk assessment.

As far as surgical treatment is considered, we tried to determine when surgery was indicated and what kind of surgical procedure was more appropriate (i.e., HT vs. TT). Based on the results of our study, less than one third (27.7\%) of indeterminate nodules harbored a malignancy, and in nearly $64 \%$ of cases, the discovered thyroid cancer was unilateral, PTMC without aggressive histological features, extracapsular invasion, or lymph node involvement. In addition, the estimated need for further surgical treatment (i.e., completion thyroidectomy) was less than $10 \%$. Therefore, HT could be considered as the treatment of choice for solitary TIR3B nodules without preoperative worrisome features, preventing overtreatment, and avoiding possible surgical complications [38-40].

On the other hand, it has to be considered that more than a half of TIR3B patients still received unnecessary surgical treatment for what was revealed to be a benign thyroid condition on final histological examination. Therefore, molecular testing has emerged as a powerful tool to improve the sensitivity and specificity of preoperative FNA cytology by enhancing risk stratification [33-35, 41]. In particular, up to $70 \%$ of thyroid cancers harbor at least one known genetic mutation and multiple markers have been studied, either alone or as a part of molecular panels. To date, the most common alteration in thyroid cancer is BRAF gene mutation, which most often occurs in papillary $(40-45 \%)$ and poorly differentiated cancers $(20-40 \%)$ and whose prevalence in indeterminate lesions varies between $15 \%$ and $40 \%$ [33, 42-44]. Another common alteration is RAS gene mutation, which is highly prevalent in follicular-patterned neoplasms, including FTCs, follicular variants of papillary thyroid carcinoma (40-50\%), and benign follicular adenomas (up to $48 \%$ ) [45-48].

In the present study, BRAF mutation in preoperative FNA cytologic samples was associated with high specificity (98.4\%) and positive predictive values (PPV) $(88.9 \%)$, although the sensitivity of the test was low (29.6\%). These results are in line with the majority of literature studies, reporting a specificity of almost $100 \%$ with relative low sensitivity rates (15-62\%) [42, 49-54]. We found only one false-positive mutation case, confirmed as adenomatous hyperplasia with underlying lymphocytic thyroiditis by surgical pathology. Surgically proven benign cases of falsepositive BRAF V600E mutation have been documented in the literature [55-57]. False-positive results can be mainly explained by the trade-off of improved sensitivity by sacrificing specificity. Indeed, the use of more sensitive diagnostic methods (e.g., pyrosequencing and the polymerase chain reaction- (PCR-) based methods), detecting the BRAF 
TABLE 4: RAS status in TIR3 population.

\begin{tabular}{|c|c|c|c|}
\hline & Number of cases & Benign & Malignant \\
\hline RAS- & 33 & 24 & 9 \\
\hline RAS+ (9 NRAS Q61R mutations, 5 NRAS Q61K mutations, 1 KRAS G12A mutation) & 15 & 8 & 7 \\
\hline & 48 & 32 & 16 \\
\hline
\end{tabular}

mutation even in small amounts of mutant DNA, results in a false-positive rate of $0.08-5.4 \%$ (depending on the method used). Moreover, benign tumors can potentially share oncogenic mutation with their malignant counterparts. Thus, the real false-positive mutation rate of BRAF testing methods in FNAC specimens may be underestimated.

On the other side, RAS mutation presented low sensitivity and low PPV (43.7\% and 46.7\%, respectively) with mediocre specificity and negative predictive values (NPV) (75.0\% and $72.7 \%$, respectively). This is once more in accordance with literature data [58] and can be easily explained considering that RAS mutation can often be present also in follicular adenomas [45-48].

All things considered, our results corroborate the hypothesis that BRAF, with its high PPV for thyroid cancer, can be used to "rule-in" malignancy and guide treatment by referring patients with mutation-positive nodules to initial TT as opposed to diagnostic HT. Additionally, considering BRAF high specificity for malignancy, the absence of this mutation in TIR3A nodules (low-risk indeterminate) may strengthen the decision for nonoperative management, as outlined by current recommendations. As concerns RAS mutation, this analysis alone is unsuitable to predict malignancy in indeterminate thyroid nodules. However, it could be useful to "rule-out" malignancy and avoid unnecessary surgery in mutation-negative indeterminate nodules.

The present study has several limitations. Being a singlecenter experience based on retrospective nonrandomized analysis, the possibility of generalizing the results is potentially limited. In addition, the sample size is small, with a disproportion between TIR3A and TIR3B patients $(24.4 \%$ and $75.6 \%$, respectively). Moreover, we recorded a relatively high incidence of PTMCs, which are currently managed through more conservative treatments considering their indolent nature among all thyroid carcinomas $[59,60]$. Last but not least, we did not consider the possibility of interoperator variability in the reading of preoperative FNA cytologic samples.

Despite these limitations, we demonstrated that the 2014 SIAPEC classification has improved diagnostic accuracy in patients with indeterminate thyroid nodules, especially with regard to malignancy rates and management strategies. Nevertheless, further studies are still required to avoid unnecessary surgical treatments. In this regard, molecular markers and immunochemistry may play a significant role in overcoming diagnostic limitations of basic cytological examination.

\section{Conclusions}

The differential diagnosis and proper treatment of indeterminate thyroid nodules remain a topic of great interest and discussion. The 2014 SIAPEC classification seems to have improved diagnostic accuracy and clinical management and its association with molecular markers may allow a better selection of surgical patients. However, further studies are required to reach a definitive agreement.

\section{Data Availability}

Data were obtained from electronic database and manual search of studies on thyroid disease. The datasets used and analyzed during the current study is available from the corresponding author on reasonable request.

\section{Ethical Approval}

This article does not contain any studies with animals performed by any of the authors. All procedures performed in this retrospective study involving human participants were in accordance with the law and the national ethical guidelines.

\section{Conflicts of Interest}

The authors declare that there are no conflicts of interest.

\section{References}

[1] S. Ezzat, D. A. Sarti, D. R. Cain, and G. D. Braunstein, "Thyroid incidentalomas. prevalence by palpation and ultrasonography," Archives of Internal Medicine, vol. 154, no. 16, pp. 1838-1840, 1994.

[2] G. H. Tan and H. Gharib, "Thyroid incidentalomas: management approaches to nonpalpable nodules discovered incidentally on thyroid imaging," Annals of Internal Medicine, vol. 126, no. 3, pp. 226-231, 1997.

[3] D. S. Dean and H. Gharib, "Epidemiology of thyroid nodules," Best Practice \& Research Clinical Endocrinology \& Metabolism, vol. 22, no. 6, pp. 901-911, 2008.

[4] S. Guth, U. Theune, J. Aberle, A. Galach, and C. M. Bamberger, "Very high prevalence of thyroid nodules detected by high frequency (13 MHz) ultrasound examination," European Journal of Clinical Investigation, vol. 39, no. 8, pp. 699-706, 2009.

[5] H. Jiang, Y. Tian, W. Yan et al., "The prevalence of thyroid nodules and an analysis of related lifestyle factors in Beijing communities," International Journal of Environmental Research and Public Health, vol. 13, no. 4, p. 442, 2016.

[6] H. Gharib and J. R. Goellner, "Fine-needle aspiration biopsy of the thyroid: an appraisal," Annals of Internal Medicine, vol. 118 , no. 4, pp. 282-289, 1993.

[7] L. Hegedüs, "The thyroid nodule," New England Journal of Medicine, vol. 351, no. 17, pp. 1764-1771, 2004.

[8] M. C. Frates, C. B. Benson, P. M. Doubilet et al., "Prevalence and distribution of carcinoma in patients with solitary and multiple thyroid nodules on sonography," The Journal of 
Clinical Endocrinology \& Metabolism, vol. 91, no. 9, pp. 3411-3417, 2006.

[9] S. I. Sherma, "Thyroid carcinoma," The Lancet, vol. 361, no. 9356, pp. 501-511, 2003.

[10] B. R. Haugen, E. K. Alexander, K. C. Bible, and G. M. Doherty, "American Thyroid Association management guidelines for adult patients with thyroid nodules and differentiated thyroid cancer: the american thyroid association guidelines task force on thyroid nodules and differentiated thyroid cancer," Thyroid, vol. 26, pp. 1-133, 2016.

[11] H. Gharib, E. Papini, J. R. Garber, and D. S. Duick, “American association of clinical endocrinologists, american college of endocrinology, and associazione medici endocrinologi medical guidelines for Clinical Practice for the Diagnosis and Management of Thyroid Nodules-2016 update," Endocrine Practice, vol. 22, no. 5, pp. 622-639, 2016.

[12] National Comprehensive Cancer Network, NCCN Clinical Practice Guidelines in Oncology (NCCN Guidelines). Thyroid Carcinoma, National Comprehensive Cancer Network, Plymouth Meeting, PA, USA, 2018, https://www.nccn.org/ professionals/physician_gls/pdf/thyroid.pdf.

[13] P. Perros, K. Boelaert, S. Colley et al., "Guidelines for the management of thyroid cancer," Clinical Endocrinology, vol. 81, pp. 1-122, 2014.

[14] L. Yassa, E. S. Cibas, C. B. Benson et al., "Long-term assessment of a multidisciplinary approach to thyroid nodule diagnostic evaluation," Cancer, vol. 111, no. 6, pp. 508-516, 2007.

[15] C.-C. C. Wang, L. Friedman, G. C. Kennedy et al., "A large multicenter correlation study of thyroid nodule cytopathology and histopathology," Thyroid, vol. 21, no. 3, pp. 243-251, 2011.

[16] M. Bongiovanni, A. Spitale, W. C. Faquin, L. Mazzucchelli, and Z. W. Baloch, "The Bethesda system for reporting thyroid cytopathology: a meta-analysis," Acta Cytologica, vol. 56, no. 4, pp. 333-339, 2012.

[17] A. M. Manning, H. Yang, M. Falciglia, J. R. Mark, and D. L. Steward, "Thyroid ultrasound-guided fine-needle aspiration cytology results: observed increase in indeterminate rate over the past decade," Otolaryngology-Head and Neck Surgery, vol. 156, no. 4, pp. 611-615, 2017.

[18] Z. W. Baloch, S. Fleisher, V. A. LiVolsi, and P. K. Gupta, "Diagnosis of ?follicular neoplasm? A gray zone in thyroid fine-needle aspiration cytology," Diagnostic Cytopathology, vol. 26, no. 1, pp. 41-44, 2002.

[19] E. S. Cibas and S. Z. Ali, "The Bethesda system for reporting thyroid cytopathology," American Journal of Clinical Pathology, vol. 132, no. 5, pp. 658-665, 2009.

[20] G. Kocjan, A. Chandra, P. A. Cross et al., "The interobserver reproducibility of thyroid fine-needle aspiration using the UK Royal College of Pathologists' classification system," American Journal of Clinical Pathology, vol. 135, no. 6, pp. 852-859, 2011.

[21] G. Fadda, F. Basolo, A. Bondi, and G. Bussolati, "Cytological classification of thyroid nodules. proposal of the SIAPEC-IAP Italian consensus working group," Pathologica, vol. 102, no. 5, pp. 405-408, 2010.

[22] F. Nardi, F. Basolo, A. Crescenzi et al., "Italian consensus for the classification and reporting of thyroid cytology," Journal of Endocrinological Investigation, vol. 37, no. 6, pp. 593-599, 2014.

[23] P. Straccia, E. D. Rossi, T. Bizzarro et al., "A meta-analytic review of the Bethesda System for Reporting Thyroid Cytopathology: has the rate of malignancy in indeterminate lesions been underestimated?" Cancer Cytopathology, vol. 123, no. 12, pp. 713-722, 2015.
[24] C. Brophy, R. Mehanna, J. McCarthy, A. Tuthill, M. S. Murphy, and P. Sheahan, "Outcome of subclassification of indeterminate (Thy-3) thyroid cytology into Thy-3a and Thy-3f," European Thyroid Journal, vol. 4, no. 4, pp. 246-251, 2015.

[25] E. Rullo, G. Minelli, D. Bosco, F. Nardi, and V. Ascoli, "Evaluation of the Italian cytological subclassification of thyroid indeterminate nodules into TIR-3A and TIR-3B: a retrospective study of 290 cases with histological correlation from a single institution," Journal of Endocrinological Investigation, vol. 41, no. 5, pp. 531-538, 2018.

[26] C. Sparano, G. Parenti, A. Cilotti et al., "Clinical impact of the new SIAPEC-IAP classification on the indeterminate category of thyroid nodules," Journal of Endocrinological Investigation, vol. 42, no. 1, pp. 1-6, 2019.

[27] P. Straccia, A. Santoro, E. D. Rossi et al., "Incidence, malignancy rates of diagnoses and cyto-histological correlations in the new Italian reporting system for thyroid cytology: an institutional experience," Cytopathology, vol. 28, no. 6, pp. 503-508, 2017.

[28] S. Ulisse, D. Bosco, F. Nardi et al., "Thyroid imaging reporting and data system score combined with the new Italian classification for thyroid cytology improves the clinical management of indeterminate nodules," International Journal of Endocrinology, vol. 2017, pp. 1-8, 2017.

[29] H. Gharib, E. Lingua::EN::Titlecase, E. Papini et al., “American Association of Clinical Endocrinologists, Associazione Medici Endocrinologi, and European Thyroid Association medical guidelines for clinical practice for the diagnosis and management of thyroid nodules: executive summary of recommendations," Journal of Endocrinological Investigation, vol. 33, no. 5, pp. 287-291, 2010.

[30] D. S. Cooper, G. M. Doherty, B. R. Haugen et al., "Revised American thyroid association management guidelines for patients with thyroid nodules and differentiated thyroid cancer," Thyroid, vol. 19, no. 11, pp. 1167-1214, 2009.

[31] J. Y. Kwak, K. H. Han, J. H. Yoon et al., "Thyroid imaging reporting and data system for US features of nodules: a step in establishing better stratification of cancer risk," Radiology, vol. 260, no. 3, pp. 892-899, 2011.

[32] A. L. Melck, L. Yip, and S. E. Carty, "The utility of BRAF testing in the management of papillary thyroid cancer," The Oncologist, vol. 15, no. 12, pp. 1285-1293, 2010.

[33] Y. E. Nikiforov, N. P. Ohori, S. P. Hodak et al., "Impact of mutational testing on the diagnosis and management of patients with cytologically indeterminate thyroid nodules: a prospective analysis of 1056 FNA samples," The Journal of Clinical Endocrinology \& Metabolism, vol. 96, no. 11, pp. 3390-3397, 2011.

[34] M. Kim and E. Alexander, "Diagnostic use of molecular markers in the evaluation of thyroid nodules," Endocrine Practice, vol. 18, no. 5, pp. 796-802, 2012.

[35] M. Nishino, "Molecular cytopathology for thyroid nodules: a review of methodology and test performance," Cancer Cytopathology, vol. 124, no. 1, pp. 14-27, 2016.

[36] R. V. Lloyd, R. Y. Osamura, G. Kloppel, and J. Rosai, World Health Organization Classification of Tumors of Endocrine Organs, IARC, Lyon, France, 4th edition, 2017.

[37] P. Trimboli, A. Crescenzi, M. Castellana, F. Giorgino, L. Giovanella, and M. Bongiovanni, "Italian consensus for the classification and reporting of thyroid cytology: the risk of malignancy between indeterminate lesions at low or high risk. A systematic review and meta-analysis," Endocrine, vol. 63, no. 3, pp. 430-438, 2019. 
[38] J. Lee, J. H. Park, C.-R. Lee, W. Y. Chung, and C. S. Park, "Long-Term outcomes of total thyroidectomy versus thyroid lobectomy for papillary thyroid microcarcinoma: comparative analysis after propensity score matching," Thyroid, vol. 23, no. 11, pp. 1408-1415, 2013.

[39] C. Dobrinja, M. Pastoricchio, M. Troian et al., "Partial thyroidectomy for papillary thyroid microcarcinoma: is completion total thyroidectomy indicated?" International Journal of Surgery, vol. 41, pp. S34-S39, 2017.

[40] G. Conzo, N. Avenia, G. L. Ansaldo et al., "Surgical treatment of thyroid follicular neoplasms: results of a retrospective analysis of a large clinical series," Endocrine, vol. 55, no. 2, pp. 530-538, 2017.

[41] W. Moses, J. Weng, I. Sansano et al., "Molecular testing for somatic mutations improves the accuracy of thyroid fineneedle aspiration biopsy," World Journal of Surgery, vol. 34, no. 11, pp. 2589-2594, 2010.

[42] Y. Cohen, E. Rosenbaum, D. P. Clark, and M. A. Zeiger, "Mutational analysis of BRAF in fine needle aspiration biopsies of the thyroid: a potential application for the preoperative assessment of thyroid nodules," Clinical Cancer Research, vol. 10, no. 8, pp. 2761-2765, 2004.

[43] I. Marchetti, F. Lessi, C. M. Mazzanti et al., "A morphomolecular diagnosis of papillary thyroid carcinoma: BRAF V600E detection as an important tool in preoperative evaluation of fine-needle aspirates," Thyroid, vol. 19, no. 8, pp. 837-842, 2009.

[44] Y. Cohen, M. Xing, E. Mambo et al., "BRAF mutation in papillary thyroid carcinoma," JNCI Journal of the National Cancer Institute, vol. 95, no. 8, pp. 625-627, 2003.

[45] S. Ezzat, L. Zheng, J. Kolenda, A. Safarian, J. L. Freeman, and S. L. Asa, "Prevalence of activating ras mutations in morphologically characterized thyroid nodules," Thyroid, vol. 6, no. 5, pp. 409-416, 1996.

[46] N. Motoi, A. Sakamoto, T. Yamochi, H. Horiuchi, T. Motoi, and R. Machinami, "Role of ras mutation in the progression of thyroid carcinoma of follicular epithelial origin," PathologyResearch and Practice, vol. 196, no. 1, pp. 1-7, 2000.

[47] M. N. Nikiforova, R. A. Lynch, P. W. Biddinger et al., "RASPoint mutations and PAX8-PPAR $\gamma$ rearrangement in thyroid tumors: evidence for distinct molecular pathways in thyroid follicular carcinoma," The Journal of Clinical Endocrinology \& Metabolism, vol. 88, no. 5, pp. 2318-2326, 2003.

[48] A. Najafian, S. Noureldine, F. Azar et al., "RAS mutations, and RET/PTC and PAX8/PPAR-gamma chromosomal rearrangements are also prevalent in benign thyroid lesions: implications thereof and a systematic review," Thyroid, vol. 27, no. 1, pp. 39-48, 2017.

[49] S. K. Kim, D.-L. Kim, H. S. Han et al., "Pyrosequencing analysis for detection of a BRAFV600E mutation in an FNAB specimen of thyroid nodules," Diagnostic Molecular Pathology, vol. 17, no. 2, pp. 118-125, 2008.

[50] A. J. Adeniran, C. Theoharis, P. Hui et al., "Reflex BRAF testing in thyroid fine-needle aspiration biopsy with equivocal and positive interpretation: a prospective study," Thyroid, vol. 21, no. 7, pp. 717-723, 2011.

[51] M. Rossi, M. Buratto, S. Bruni et al., "Role of ultrasonographic/clinical profile, cytology, and BRAF V600E mutation evaluation in thyroid nodule screening for malignancy: a prospective study," The Journal of Clinical Endocrinology \& Metabolism, vol. 97, no. 7, pp. 2354-2361, 2012.

[52] D. A. Kleiman, M. J. Sporn, T. Beninato et al., "Preoperative BRAF (V600E) mutation screening is unlikely to alter initial surgical treatment of patients with indeterminate thyroid nodules," Cancer, vol. 119, no. 8, pp. 1495-1502, 2013.

[53] A. Najafian and M. A. Zeiger, "Role of molecular diagnostic markers in the management of indeterminate and suspicious thyroid nodules," International Journal of Endocrine Oncology, vol. 1, no. 1, pp. 49-57, 2014.

[54] A. Kowalska, A. Kowalik, I. Pałyga et al., "Przydatność określania obecności mutacji BRAF V600E w biopsji aspiracyjnej celowanej cienkoigłowej $\mathrm{w}$ zmianach niezdeterminowanych," Endokrynologia Polska, vol. 67, no. 1, pp. 41-47, 2016.

[55] S. K. Kim, T. S. Hwang, Y. B. Yoo et al., "Surgical results of thyroid nodules according to a management guideline based on the BRAFV600EMutation status," The Journal of Clinical Endocrinology \& Metabolism, vol. 96, no. 3, pp. 658-664, 2011.

[56] S.-Y. Kim, E.-K. Kim, J. Y. Kwak, H. J. Moon, and J. H. Yoon, "What to do with thyroid nodules showing benign cytology and BRAFV600E mutation? A study based on clinical and radiologic features using a highly sensitive analytic method," Surgery, vol. 157, no. 2, pp. 354-361, 2015.

[57] C. K. Zhao, J. Y. Zheng, L. P. Sun, R. Y. Xu, Q. Wei, and H. X. Xu, "BRAF V600E mutation analysis in fine-needle aspiration cytology specimens for diagnosis of thyroid nodules: the influence of false-positive and false-negative results," Cancer Medicine, vol. 8, no. 12, pp. 5577-5589, 2019.

[58] W. Clinkscales, A. Ong, S. Nguyen, E. E. Harruff, and M. B. Gillespie, "Diagnostic value of RAS mutations in indeterminate thyroid nodules: systematic review and metaanalysis," Otolaryngology-Head and Neck Surgery, vol. 156, no. 3, pp. 472-479, 2017.

[59] Y. Ito, T. Uruno, K. Nakano et al., “An observation trial without surgical treatment in patients with papillary microcarcinoma of the thyroid," Thyroid, vol. 13, no. 4, pp. 381-387, 2003.

[60] Y. Ito and A. Miyauchi, "A therapeutic strategy for incidentally detected papillary microcarcinoma of the thyroid," Nature Clinical Practice Endocrinology \& Metabolism, vol. 3, no. 3, pp. 240-248, 2007. 